\title{
Contourlet Transformation for Text Hiding in HSV Color Image
}

\author{
KHALIL IBRAHIM ALSAIF ${ }^{1}$ and MEAAD M. SALIH ${ }^{2}$ \\ ${ }^{12}$ Dept of Computer Science, College of Computer \& Mathematic Science-Mosul Univ./ IRAQ \\ E-mail: 'skhalil_alsaif@hotmail.com, ${ }^{2}$ meaad_muhammad@yahoo.com²
}

\begin{abstract}
Texts hiding in digital image have recently received quite a bit of attention because it is very important in invisible communication. This paper presents a new data hiding technique for embedding text data in images represented in HSV color space using (NSCT). The text data is first converted to ASCII format and then represented in binary form and then, added to contourlet coefficients. A high frequency directional pass band from of the contourlet transform is selected for data embedding. Image features like Peak Signal to Noise Ratio (PSNR). The proposed method show that we could successfully embed data in cover_images and extract it with the average embedding capacity of (1/16) bits per pixel without any error. High capacity can be achieved using this method according to block size.
\end{abstract}

Keywords: $H S V_{-}$color image, steganography, contourlet transform, stego_image.

\section{INTRODUCTION}

Data hiding involves hiding secret messages into the cover object with a slight distortion. It represents various applications, for example, copyright protection (watermarking), secret communication (steganography), image authentication, and so on. Though various applications have different requirements, data hiding has two fundamentals. First, the embedding distortion should be minimized. As far as security is concerned, the embedding changes in cover object should not be conspicuous. Secondly, the hiding capacity should suffice to embed a reasonable amount of embedded messages. However, a tradeoff between the embedding distortion and the hiding capacity is inevitable. Obviously, the goal of data hiding is to design schemes with high hiding capacity but low embedding distortion [1].

Steganography is a technique for covert information hide the existence of the message from third persons [2]. All steganographic algorithms have to comply with a few basic requirements. The most important requirement is that a steganographic algorithm has to be imperceptible [3] . The aim of the steganography methods is to communicate securely in a completely undetectable manner. The current approaches in steganography domain could be divided into two categories of spatial and transformation domains. In spatial domain, there are many strategies of image steganography based on manipulating the least significant bit using direct replacing of least significant bit levels with the bits of secret image [4]. In transform domain, secret message is embedded in the coefficients of cover media in frequency domain. In transform domain cosine, wavelet etc are used as transforms [5]. Steganography in the frequency domain is one of the growing research areas in recent years because of its capability of providing robustness to attacks and posing a real challenge to anybody trying to discover and decode hidden messages [6]. In proposed method we embedding text in HSV color images, storing data in blocks of coefficients in high frequency subbands, but we use Nonsampled Counterlet Transform (NSCT). One of the advantages of counterlet transform is the existence of linearly independent subbands. This issue decreases the possibility of detection of the stegano image by steganalysis algorithms [5].

\section{RELATED WORK}

In 2002, Chin-Chen Chang, Tung-Shou Chen and Lou-Zo Chung presented in their research "A steganographic method based upon JPEG and quantization table modification", a novel steganographic method based on joint photographic 
expertgroup (JPEG). The proposed method modifies the quantization table first. Next, the secret message is hidden in the cover-image with its middle-frequency of the quantized DCT coeficients modified. Proposed technique provides acceptable image quality and a large message capacity [7].

Wen-Chao Yang, Ling-Hwei Chen in 2008, in their research "A Novel Steganography Method via Various Animation Effects in Powerpoint Files", a novel steganography method that hides message via various animation effects in a PowerPoint file is proposed. The proposed method uses different animation effects to stand for different characters, thus a codebook is designed to record the corresponding between the animation effects and characters. The proposed method contains two parts, one is the embedding process and the other is extracting process. Both processes use the same animation codebook as a look-up table. This method does not distort the PowerPoint file [8].

In 2009, Sudeep P.V. \& K.A. Navas \&Sheeba V.S. in their research "A Novel Datahiding Method In Spatial Domain " proposed technique hides ASCII characters in tle least significant digits (LSD) of the image. This investigation mainly focuses on enhancing the capacity of data hiding in medical images ,user has the choice for selecting the number of LSDs of the pixel magitude. This method achieving enormous capacity without sacrificing the visual quality of the images. $1 \mathrm{MB}$ data can be embedded in $512 \times 512$ in gray scale image [9].

Dipti Kapoor Sarmah \& Neha Bajpai presented in 2010 in their research "Proposed System for Data Hiding Using Cryptography and Steganography " they are developing a system where they develop a new technique in which Cryptography and Steganography are used as integrated part along with newly developed enhanced security module. In Cryptography they are using AES algorithm to encrypt a message and a part of the message is hidden in DCT of an image; remaining part of the message is used to generate two secret keys which make this system highly secured [10].

S. Suma Christal Mary presented in her research " Improved Protection In Video Steganography Used Compressed Video Bitstreams " in 2010, a new method for the real-time hiding of information used in compressed video bitstreams . A new compressed video secure steganography (CVSS) algorithm is proposed. In this algorithm, embedding and detection operations are both executed entirely in the compressed domain, with no need for the decompression process. The new criteria employing statistical invisibility of contiguous frames is used to adjust the embedding strategy and capacity, which increases the security of proposed algorithm. Therefore, the collusion resistant properties are obtained [11].

In 2011, Malini Mohan \& Anurenjan. P. R in their research "A Novel Data Hiding Method in Image using Contourlet Transform " , they applied an encryption algorithm which provides additional security. A digit of data is embedded by modifying the least significant digit of a contourlet coefficient. A high frequency directional pass band from the contourlet transform is selected for data embedding. High capacity can be achieved using this method. The performance of this proposed method in other transform domains like DCT and wavelet transform alse evaluated and compared with that in contourlet domain [3] .

In 2012 , Nitin Jain, Sachin Meshram, Shikha Dubey presented In their paper " Image Steganography Using LSB and Edge - Detection Technique ", search how the edges of the images can be used to hiding text message in Steganography. For that gray image has been presented. They tried to find dark places of gray image (black) by converting the original image to binary image for labeling each object of image by considering on 8 pixel connectivity. Then these images have been converted to RGB image in order to find dark places. In the final stage each 8 pixels of dark places has been considered as a byte and binary value of each character has been put in low bit of each byte that was created manually by dark places pixels for increasing security of the main way of least Significant bit steganography[12] .

In 2012 , R. Thamaraiselvan and A. Saradha proposed a novel approach of text steganography method in their research " A Novel Approach Of Hybrid Method Of Hiding The Text Information Using Steganography " by using interword spacing and interparagraph spacing as a hybrid method. Based on the length of the secret message, the proposed method provides dynamic generated stegotext with six options of maximum capacity [13].

\section{DESCRIPTION OF HSI MODEL}

The HSI color model (abbreviation of hue, saturation and intensity), as it was mentioned before, is standard of color image processing. The idea of this model implementation is not so easy; usually this standard can be represented graphically in hue, saturation, and intensity in 3 dimensional spaces as shown in Figure 1 [14]. 


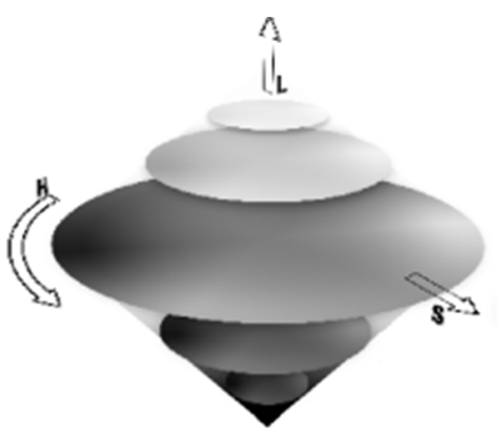

Fig. 1. Double Cone Model of HSV Color Space

\section{CONTORELET TRANSFORM}

The Contourlet transform was proposed by M. N. Do and M. Vetterli. The contourlet transform provides a multi scale and multi-directional representation of an image. It consists of a double filter bank structure for obtaining sparse expansions for typical images having smooth contours. In this double filter bank, the Laplacian pyramid (LP) is first used to capture the point discontinuities, and then followed by a directional filter bank (DFB) to link point discontinuities into linear structures. The required number of directions can be specified by the user. Since contourlets gives more edges, it is more suitable for data hiding applications as more data can be hidden in the high frequency regions without perceptually distorting the original image. The overall results in an image expansion using basic elements like contour segments, and, thus, are named contourlets[3] .

\subsection{Nonsubsampled Contourlet Transform (NSCT)}

The nonsubsampled contourlet transform is a new image decomposition scheme introduced by Arthur L.Cunha, Jianping Zhou and Minh N.Do [14] NSCT is more effective in representing smooth contours in different directions of in an image than contourlet transform and discrete wavelet transform. The NSCT is fully shift invariant, Multi scale and multi direction expansion that has a fast implementation. The NSCT exhibits similar sub band decomposition as that of contourlets, but without down samplers and up samplers in it. Because of its redundancy, the filter design problem of nonsubsampled contourlet is much less constrained than that of contourlet The NSCT is constructed by combining nonsubsampled pyramids and nonsubsampled directional filter bank as shown in Figure (2) .The nonsubsampled pyramid structure results the multi scale property and nonsubsampled directional filter bank results the directional property.figure (3) show an example of NSCT .

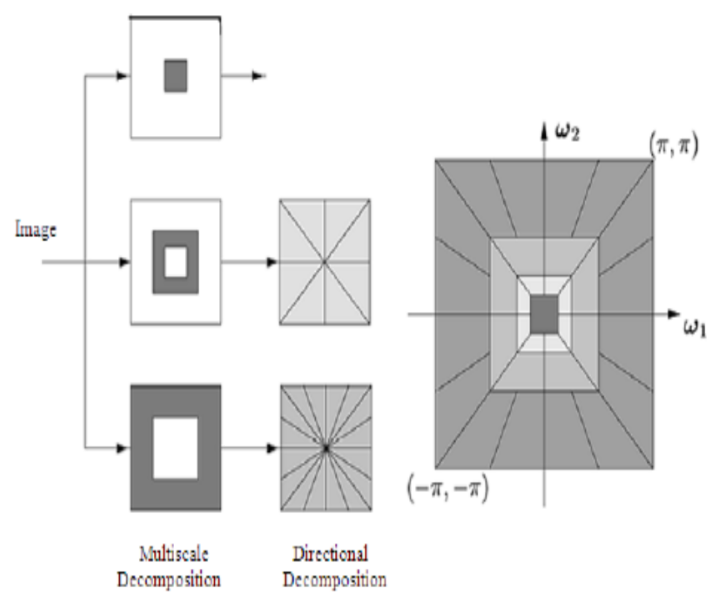

Fig. 2. The nonsubsampled contourlet transform a) nonsubsampled filter bank structure that implements the NSCT. b) Idealized frequency partitioning obtained with NSCT

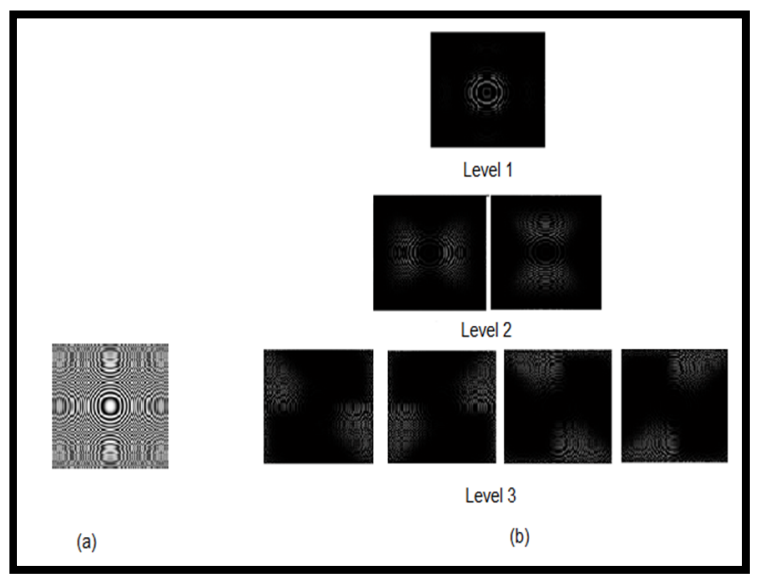

Fig. 3. The contourlet transform in three levels: a) orginal image b) decomposed image

\subsubsection{Nonsubsampled pyramids}

The nonsubsampled pyramid is a two channel nonsubsampled filter bank as shown in Figure 4(a). The $\mathrm{HO}(\mathrm{z})$ is the low pass filter and one then sets $\mathrm{H} 1(\mathrm{z})=1-\mathrm{H} 0(\mathrm{z})$ and corresponding synthesis filters $\mathrm{G} 0(\mathrm{z})=\mathrm{G} 1(\mathrm{z})=1$. The perfect reconstruction condition is given by Bezout identity[14],[15].

H0 (z) G0 (z) +H1 (Z) G1 (Z) =1 _. 1 


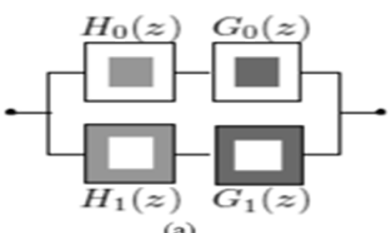

(a)

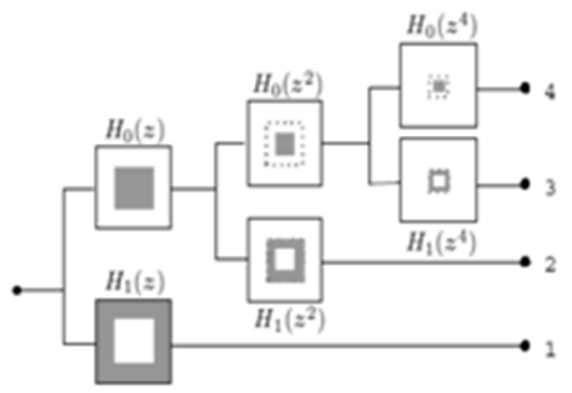

(b)

Fig. 4. Nonsubsampled pyramidal filters (a). Ideal frequency response of nonsubsampled pyramidal filter (b).The cascading analysis of three stages nonsubsampled pyramid by iteration of two channels nonsubsampled filter banks.

Multi scale decomposition is achieved from nonsubsampled pyramids by iterating the nonsubsampled filter banks. The next level decomposition is achieved by up sampling all filters by 2 in both dimensions. The complexity of filtering is constant whether the filtering is with $\mathrm{H}(\mathrm{z})$ or an up sampled filter $\mathrm{H}(\mathrm{z} \mathrm{m})$ computed using " a trous " algorithm The cascading of three stage analysis part is shown in Figure (4) b .

\subsubsection{Nonsubsampled directional filter banks}

The directional filter bank (DFB is constructed from the combination of critically-sampled twochannel fan filter banks and resampling operations. The outcome of this DFB is a tree structured filter bank splitting the 2-D frequency plane into wedges. The nonsubsampled directional filter bank which is shift invariant is constructed by eliminating the down and up samplers in the DFB.The ideal frequency response of nonsubsampled filter banks is shown in Figure (5) a .

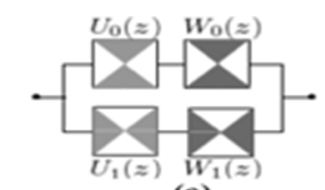

(a)

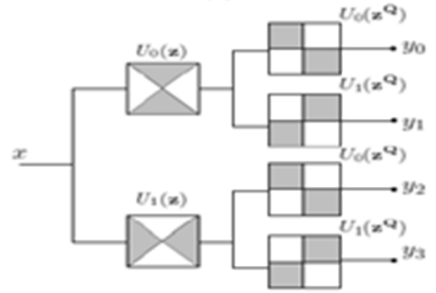

(b)

Fig. 5. Nonsubsampled directional filter bank (a) idealized frequency response of nonsubsampled directional filter bank.(b) The analysis part of an iterated nonsubsampled directional bank.

To obtain multi directional decomposition, the nonsubsampled DFBs are iterated. To obtain the next level decomposition, all filters are up sampled by a quincunx matrix given by [14],[15]

$$
Q=\left(\begin{array}{rr}
1 & 1 \\
1 & -1
\end{array}\right)
$$

2

The analysis part of an iterated nonsubsampled filter bank is shown in Figure 5(b).

\section{AIM OF THE PAPER}

New technique will be achieved for data hiding depend on dividing one of the contourlet coefficients to blocks and embedded the message bits in its center.

\section{PROPOSED ALLGORITHM}

Figure 6 show the block diagram of proposed algorithm. The algorithm contain two phase the first one handle the embedding data inside the image while the second phase cover the extract the embedded data ,this figure placed at the end of this paper . 


\subsection{Proposed Embedding Algorithm}

The embedding process is done in the following steps at the sender side:

Step 1: Select one of HSV color space slides as a cover image then, decomposed using (NSCT). So the coefficients will be low/high frequency with same size as the original one . Looking for the best coefficient for embedding (high pass subbands are chosen for embedding).

Step 2: The embedded message will be converted to ASCII code, so that it will be represented in binary format .

Step 3: embedding process steps :

a) The identified high pass subband divided into blocks, and prepared that each block will hold one bit from the secret message.

b) each bit ( 0 or 1 ) of the message to be embedded by adding it's value to the value of block center.

$\mathrm{m}=\mathrm{d}+\mathrm{V} \_$center ............... 3

where $\mathbf{d}$ is the bit to be embedded and V_center is the value of center of block .The process is continue until the whole data is embedded .

Step 4: reconstruct the image from the processed coefficients to obtain stego_slide ( $\mathrm{H}, \mathrm{S}$ or $\mathrm{V})$ and then rebuild the image from three slides( one of them stego_slide ) to obtain stego_image in HSV color space (cover image with secret data).

Step 5: embed the block size and threshold value in stego_image by replacing position $(1,1)$ and $(1,2)$ of stego_image with values of block size and threshold value respectively.

\subsection{Proposed Extracting Algorithm}

The steganography method of the proposed system is blind. Extracting process is done in the following steps at the recipient side:

Step 1: extract block size and threshold value from stego_image which were embedded in it.

Step 2: The recipient received image (stego_image) and then, select identified slide (slide_stego) and decomposed it using ( NSCT ) into high frequency subbands and identified equivalent high frequency subband S_stego .

Step 3: identified equivalent high frequency subband S_cover from decomposed cover ;

Step 4: evaluate the different between S_cover and S_stego to get the difference matrix (D_matrix) .
Step 5 : divided ( D_matrix) in step4 into blocks and then treat center of each block by comparing it with threshold value (V_center). The process is continued until the whole embedded data will extracted .

Step 6: the obtained binary sequence will be converted to ASCII code to get the secret message.

\subsection{Applied Example}

Figure 6 show the secret message (1032 char) that hiding in cover Image in HSV color space ( peppers $256 \times 256)$ shown in figure $(7-a)$ after converted it to binary sequence to obtain the stego_image in figure (7-b).

\begin{tabular}{|l}
\hline we using contourlet a new data hiding \\
technique for embedding text data in \\
image............................................. $\begin{array}{r}\text { for } \\
\text { embedding text data in image using } \\
\text { contourle. }\end{array}$. \\
\hline
\end{tabular}

Fig. 6. Tthe secret message

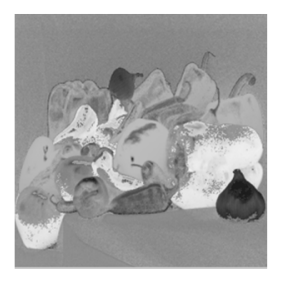

(a)

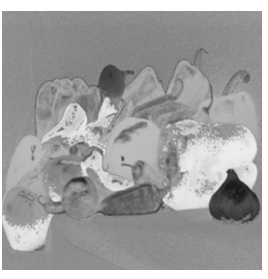

(b)
Fig. 7. a) Cover image, b) Stego_image

\section{RESULT AND DISCUSSION}

To evaluate the performance of the proposed embedding algorithm experiment was conducted using color images with different size. Simulations were done using MATLAB.

The selected slide of image in HSV color space is composed to level two using NSCT. The result contains a low_ pass band and four high _ pass subbands. One of the subbands is chosen for data embedding related to it's features. Proposed allgorithm tested using different block sizes to embed data with different threshold values [0.0005 $\leq$ Threshold $\leq 0.2]$ for extraction. Figure 8 show that embedding process with block size $=3 \times 3$ give high capacity for embedding data with less error than other sizes $(4 \times 4-11 \times 11)$. Also was seen that the extraction process depends on threshold value, figure (9) show threshold value and Error rate percent in extracted data according to that threshold .It is clear that less Error appear on threshold value (0.01 and 0.05) for all block sizes. 


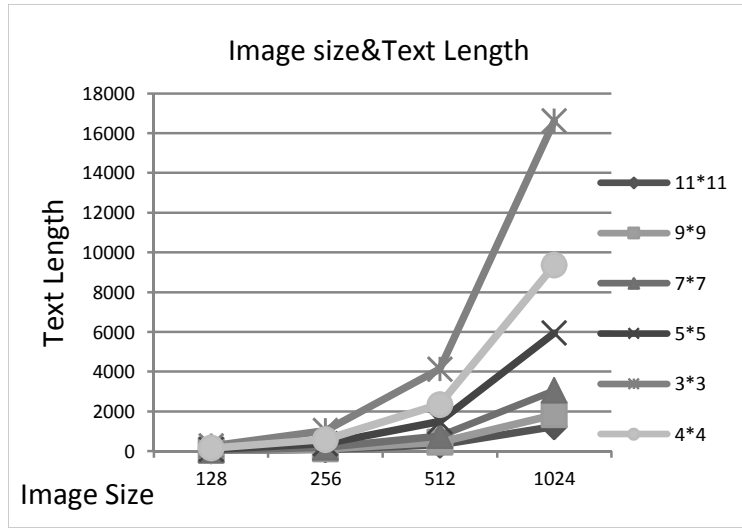

Fig. 8. Show embedding capacity according to block size

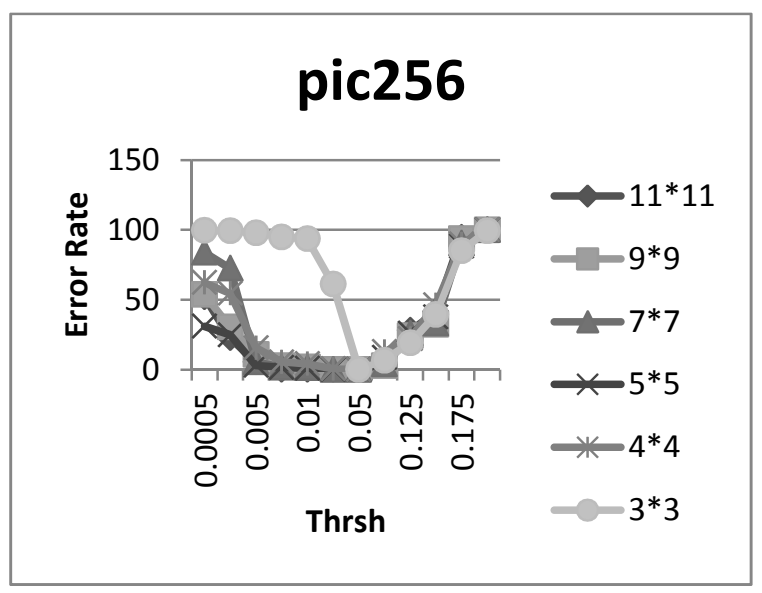

Fig. 9. Show error rate in extracted data from stego image $512 \times 512$

Image features like PSNR, MSE, Correlation are also analysed between orginal image and retrieval one . PSNR stands for peak signal to noise ratio, which is a measure for image quality perpose. The PSNR is most commonly used as a measure of quality of reconstruction in image compression etc. It gives the ratio between the maximum possible power of a signal and the power of corrupting noise that affects the fidelity of its representation. It is most easily defined using the mean squared error (MSE) which computed for two monochrome images $\mathrm{I}$ and $\mathrm{K}$ where one of them is considered as a noisy approximation of the other. $\operatorname{Equ}(4,5)$ shows the MSE and PSNR evaluation .

MSE $=\frac{1}{m n} \sum_{i=1}^{m} \sum_{j=1}^{n}\|I(i, j)-K(i, j)\|$ ...4

$P S N R=10 \times \log \left(\frac{255^{2}}{M S E}\right)$
Correlation coefficient between cover image and stego image will be calculated by equ(6) to see how much the retrieval image close to the orginal one.

$$
\operatorname{Corr} 2=\frac{\sum_{m} \sum_{n}\left(A_{m n}-\bar{A}\right)\left(B_{m n}-\bar{B}\right)}{\sqrt{\left(\sum_{m} \sum_{n}\left(A_{m n}-\bar{A}\right)^{2}\right)\left(\sum_{m} \sum_{n}\left(B_{m n}-\bar{B}\right)^{2}\right)}} \ldots \ldots \ldots \ldots \ldots \ldots \ldots . .6
$$

Where $\bar{A}, \bar{B}=$ mean of the value in (A) and (B) computes the mean of the values in A and B respectively.

Figure 10 shows the variation of PSNR and MSE and Correlation for different volume of embedded data.

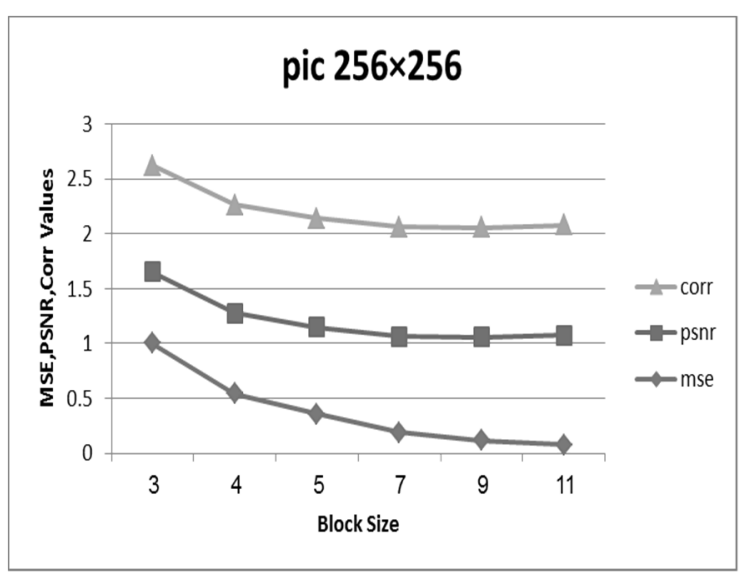

Fig. 10. MSE \& PSNR \& Correlation

\section{CONCLUSION}

From the applied example on the proposed idea of using the contourlet coefficient, gives that hiding data in the coefficients of the contourlet gaves a robust technique for high security .

High performance of data embedded ,segmentation of the cover image in same block size achieved to reach, which goes to less error rate between the original message and the retrieved one, and also high capacity when the cover image was segmented on $4 \times 4$ block size .

In addition to the high performance on retrieving the message, the cover message was tested by evaluating the correlation factor ,MSE ,PSNR between the original cover and stego_cover ,although the effect of the cover size was processed to show it's effect on the message length and the relation between them. 


\section{REFERENCES}

[1] Tai W. and Chang C. , (2009 ). "Data ahiding based On VQ Compressed Images Using Hamming Codes And Declustering ", International Journal of Innovative Computing, Information and Control, Vol 5, No 7, ISSN 1349-4198 .

[2] ZAMANI M . ,(2010) . " Genetic Based Substitution techniques For Audio Steganography ",PHD thesis , University Teknologi Malaysia DECEMBER .

[3] Malini Mohan and Anurenjan P.R (2011). "A New Algorithm for Data Hiding in Images using Contourlet Transform", 978-1-42449477-4/11/ IEEE .

[4] Saeed Masaebi et al., (2012 ). "A New Approach for Image Hiding Based on Contourlet Transform ",International Journal of Electrical and Computer Engineering (IJECE)Vol.2, No.5,ISSN: 2088-8708 .

[5] Rubab S. and Younus M.,(2012) ," Improved Image Steganography Technique for Colored Images using Huffman Encoding with Symlet Wavelets ", IJCSI International Journal of Computer Science Issues, Vol. 9, Issue 2, No 1,ISSN (Online): 1694-0814 .

[6] Sushil Kumar and S.K.Muttoo (2011). "Steganography based on Contourlet Transform " ,(IJCSIS) International Journal of Computer Science and Information Security, Vol. 9, No. 6 .

[7] Chang C. et al., (2002) . "A steganographic method based upon JPEG and quantization table modification", Intelligent Multimedia Computing and Networking Atlantic City, NJ, USA, Vol 141.

[8] YANG W. and CHEN L. ,(2008)." A Novel Steganography Method Via Various Animation Effects In Powerpoint Files ", Proceedings of the Seventh International Conference on Machine Learning and Cybernetics, Kunming, 12-15.

[9] Sudeep P.V. et al., (2009). “ A Novel Datahiding Method in Spatial Domain", 10th National Conference on Technological Trends (NCTT09).

[10] Sarmah D. and Bajpai N. , (2010) ."Proposed System for Data Hiding Using Cryptography and Steganography “ ,International Journal of Computer Applications (0975 - 8887) Volume 8- No.9.

[11] Suma Christal Mary S.,(2010) ," Improved Protection In Video Steganography Used Compressed Video Bitstreams ", IJCSE)
International Journal on Computer Science and Engineering Vol. 02, No. 03.

[12] Jain N. , Meshram S. , Dubey S., (2012)," Image Steganography Using LSB and Edge Detection Technique ", International Journal of Soft Computing and Engineering (IJSCE) ISSN: 2231-2307, Volume-2, Issue-3 .pp.217222.

[13] Thamaraiselvan R., Saradha A.,(2012)." A Novel Approach Of Hybrid Method Of Hiding The Text Information Using Steganography ", International Journal of Computer and Electronics Research, Volume 1, Issue 1, ISSN: 2778-5795 .

[14]Jianping Zhou; Cunha, A.L, M.N.Do, "Nonsubsampled contourlet transform construction and application in enhancement" ,IEEE Trans. Image Proc Sept, 2005.

[15]M. N. Do et al., (2005), "Nonsubsampled contourlet transform: filter design and applications in denoising" EEE international conference on image processing. 
K. I. Alsaif and M. M. Salih / International Journal of Computer Networks and Communications Security, 1 (4), September 2013

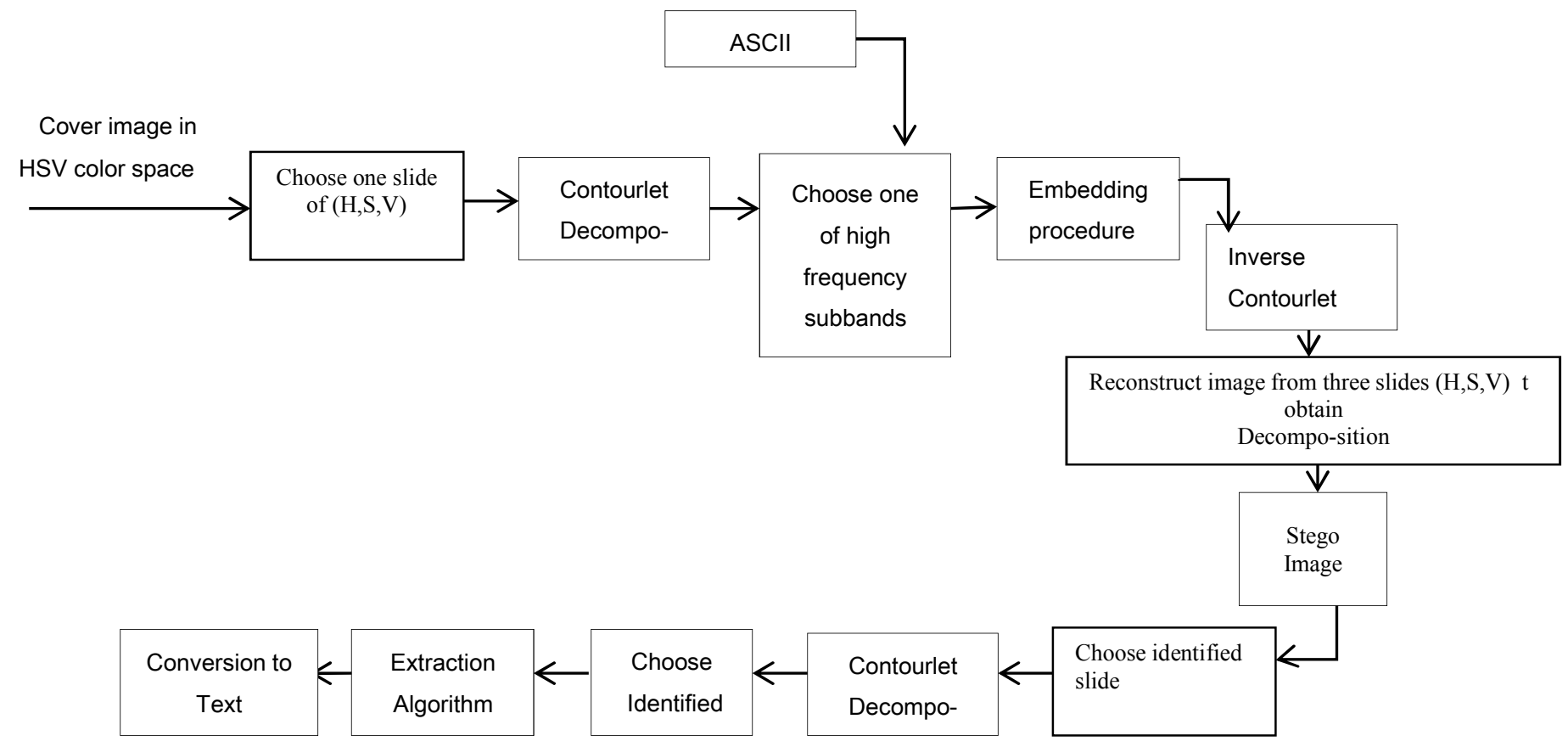

Fig. 6. Show the Block Diagram Of Proposed System 